\title{
Symposium
}

\section{Vom empiristischen Humanismus zum partizipativen Sozialismus}

Symposiumsbeitrag zu: Thomas Piketty, Kapital und Ideologie. München: C.H. Beck 2020, 1312 S., gb., 39,95€

Besprochen von Prof. Dr. Jakob Kapeller: Institut für Sozioökonomie, Universität Duisburg-Essen und Forschungsinstitut für die Gesamtanalyse der Wirtschaft, Johannes Kepler Universität LinzDuisburg-Essen, E-Mail: jakob.kapeller@uni-due.de und Prof. Dr. Miriam Rehm: Institut für Sozioökonomie, Universität Duisburg-Essen und Department für Sozioökonomie, Wirtschaftsuniversität Wien, E-Mail: miriam.rehm@uni-due.de

https://doi.org/10.1515/srsr-2021-0004

Schlüsselwörter: Verteilung, Ungleichheit, Wirtschafts- und Sozialgeschichte, Sozialismus

„Geschichte ist Resultat von Krisen, sie ist nichts, was im Voraus in den Büchern geschrieben steht.“

(Piketty: 1187)

\section{Kapital und Ideologie: Intention und Inhalt}

Thomas Piketty hat mit „Kapital und Ideologie“ erneut eine intellektuelle Tour de Force vorgelegt, die konsequent an sein Erstlingswerk „Kapital im 21. Jahrhundert“ anschließt, dem Leser bzw. der Leserin allerdings mit über 1.300 Seiten in der deutschen Fassung einiges an Durchhaltevermögen abverlangt. Piketty bleibt dabei sowohl seinem Stil als auch seinem Ziel treu: Akribische Datensammlungen untermauern institutionell-historische Analysen, die so nuanciert, umsichtig und vielschichtig formuliert sind, dass wenig Raum für fundamentalen Widerspruch bleibt. Piketty zieht dabei politische Schlussfolgerungen aus seiner historischen Analyse, die letztlich zu einem Bündel radikal-reformistischer Politikvorschläge führen, die von dem Ziel getragen sind, die Verteilungsfrage im 21. Jahrhundert auf demokratischer Grundlage neu zu verhandeln. Ob der umfassende, teilweise etwas repetitive, jedoch stets flüssig zu lesende Text hierzu tatsächlich Impulse zu geben vermag, kann nur die Zeit zeigen. Jedenfalls handelt es sich bei „Kapital und Ideologie“ um einen neuerlichen Versuch, die grundsätzlichen Koordinaten 
des Diskurses zu Ungleichheitsfragen weiter zu verschieben - so, wie es Piketty bereits mit „Kapital im 21. Jahrhundert“ gelungen ist.

Sein Ansatz entspringt einer aufklärerischen Grundhaltung, die sich bemüht zu zeigen, dass Eigentum etwas ist, das sich selbst reproduziert und Gesellschaften dabei zugleich spaltet, prägt und grundlegend strukturiert. Eigentum ist dabei kulturell und politisch festgelegt, und kann somit sowohl gestärkt als auch hinterfragt werden - Verteilungsfragen sind also politisch gestaltbar, ja, sie repräsentieren für Piketty sogar die zentrale politische Gestaltungsdimension. Die Verteilungsergebnisse, die wir in Gegenwart und Geschichte beobachten, sind für Piketty in „Kapital und Ideologie“ nicht durch die abstrakten ökonomischen Gesetzmäßigkeiten der Grenzproduktivität determiniert (die in der Standardökonomie und auch noch in „Kapital im 21. Jahrhundert“ eine zentrale Rolle spielen), sondern vielmehr durch den selbstreproduzierenden Charakter des Vermögens bestimmt. Für die menschliche Historie kennzeichnend ist dabei die Interaktion zwischen der sich selbst verstärkenden Vermögensungleichheit (also dem Umstand, dass große Vermögen schneller wachsen als kleine) und den menschengemachten Regeln, Institutionen und Ideologien, die den Umgang mit Eigentum in einer Gesellschaft regulieren und legitimieren.

Mit dieser Denkweise verlässt Piketty den heute maßgeblichen standardökonomischen Denkrahmen und kehrt zurück zum Verständnis jener ökonomischen Klassiker, die die Ökonomie vornehmlich als Gesellschaftswissenschaft und die Verteilung als „alleinige Sache der menschlichen Institutionen“ (Mill, 1848: II.1.1-2; Übersetzung JK/MR) begriffen haben. Wiederholt verweist Piketty darauf, dass Eigentumsverhältnisse politisch gesetzt sind - und dass sich diese Setzungen im Rahmen historischer Umbrüche rasant verändern können. Diese Veränderungen werden nach Piketty vornehmlich durch vorherrschende theoretische und moralische Ideen gestaltet - eine Ansicht, die er unter anderem mit John Maynard Keynes und Friedrich August von Hayek teilt, die beide ebenfalls die Macht der Ideen als entscheidend für die wirtschaftspolitische Entwicklung ansahen.

Pikettys Hauptanliegen ist die Herstellung größerer ökonomischer Gleichheit, die für ihn zwar einen positiven moralischen Eigenwert hat, aber kein Selbstzweck bleibt. Nur größere ökonomische Gleichheit erlaubt es, individuelle Freiheit und sozialen Frieden langfristig zu sichern und zugleich zentrale Zukunftsherausforderungen des 21. Jahrhunderts - wie die Bewältigung des Klimawandels, die Schaffung föderaler und (möglichst) globaler demokratischer Strukturen oder die Nutzung neuer Technologien im Sinne der Sicherung und des Ausbaus der Menschenrechte - gezielt zu adressieren. Zudem folgt Piketty einer Theorie der „langen Wellen“ der Vermögensungleichheit (z. B. Milanovic, 2016; van Bavel, 2016; Scheidel, 2017) - eine immerwährend steigende Konzentration 
der Vermögen ist gesellschaftlich wie politisch nicht auf Dauer haltbar -, und es gilt daher, die brutalen Verwerfungen zu vermeiden, die sowohl mit der persistenten Unterdrückung und Exklusion subalterner Schichten als auch mit gewaltgeleiteten Reduktionen der Ungleichheit einhergehen.

Obgleich sich Piketty immer wieder bemüht, intellektuelle Distanz zu Marx zu signalisieren, erzählen doch beide eine an vielen Stellen sehr ähnliche Geschichte der Menschheit: für beide ist die Eigentumsfrage konstitutiv für die jeweilige Gesellschaftsordnung und politische Veränderungen zentral für die Umwälzung der aus Eigentumsverhältnissen entstehenden Machtasymmetrien. Beide vertreten die These einer endogenen Akkumulation und Konzentration, wobei Marx auf die „Produktionsmittel“ im engeren Sinne abstellt und somit stärker theoretisch argumentiert, während Piketty einen breiteren, datengetriebenen Vermögensbegriff verwendet und für die Widersprüche, die etwa die Kapitalkontroverse (Cohen/ Harcourt, 2003) aufgedeckt hat, wenig Geduld aufbringt. Für beide ist die Geschichte der Menschheit eine Geschichte von „Ungleichheitsregimen“ - ein Begriff, den Piketty an mancher Stelle sogar als Synonym zur „Gesellschaft“ setzt -, in denen sich Eigentumsverhältnisse meist pfadabhängig nach dem Matthäusprinzip entwickeln (,wer hat, dem wird gegeben“) und Ungleichheiten damit außergewöhnlich markant und persistent sind.

Ungeachtet dieser zentralen Gemeinsamkeiten gibt es auch eine Reihe wichtiger Unterschiede: im Vergleich zu Marx betont Piketty stärker die dominante Rolle von Ideen und Ideologien sowie Momente historischer Kontingenz. Auch wenn er seinen Blick auf die Basis von Eigentum weitet, scheut Piketty im Gegensatz zu Marx doch davor zurück, den kapitalistischen Wettbewerb (der durch Gewinnerzielungs- und Wachstumszwang sowie Kosteneinsparungen bei den Arbeitskräften gekennzeichnet ist) grundlegend in Frage zu stellen. Stattdessen fokussiert er auf eine radikal gedachte Variante steuerlicher Umverteilung. Piketty wendet sich auch gegen ein - aus seiner Sicht zu simples - Verständnis von Gesellschaften als quasi-dichotome „Klassengesellschaften“. Sein Verständnis von Verteilung ist dabei viel weniger „theoriegeladen“ als jenes von Marx: Piketty geht nicht von einem „funktionalen“ Verständnis der Verteilung aus, das Arbeit und Kapital gegenüberstellt, sondern von einem Zugang, der auf der Betrachtung der personalen Verteilung beruht, und sich mit der Gegenüberstellung von „arm“ und „reich“, von „besitzlos“ und „,vermögend“ begnügt. Diese Herangehensweise Pikettys macht für die Diskussion der alten Ständestaaten, die eher dem dichotomen Ideal entsprechen, nur wenig Unterschied, während es für die Diskussion der Situation moderner Gesellschaften durchaus lehrreiche Einsichten bereithält. Dies sticht vor allem im vierten Teil des Buches positiv heraus, der eine politikwissenschaftliche Analyse der Entwicklung der Wählerbasis sozialdemokratischer Parteien enthält, die Bedrohung durch „sozial-nativistische“ 
Bewegungen zu erklären versucht und einen Gegenentwurf in Form eines egalitär-internationalistischen „partizipativen Sozialismus“ präsentiert.

\section{2. „Kapital und Ideologie“: Methodische Grundlagen und theoretische Einsichten}

Wenn also die Verteilung von Eigentum zwar einem systemimmanenten Trend zur Konzentration unterworfen ist, diese Verteilungsergebnisse aber immer auch sozial eingebettet und politisch gerahmt sind, dann können Gesellschaften mit Rückgriff auf das vorherrschende Ungleichheitsregime beschrieben werden. Dabei meint ein Ungleichheitsregime nicht nur eine bestimmte Verteilungssituation, sondern inkludiert stets auch eine Ideologie oder Erzählung, die diese Verteilungssituation rechtfertigt. So wurde etwa die „trifunktionale Ordnung“ der alten Ständestaaten, in der im Wesentlichen nur Adel und Klerus, ein Bruchteil der Bevölkerung, nennenswerten Besitz aufwiesen, durch die Idee einer ,göttlich gewollten Ordnung“ oder die Vorstellung des „Lehensversprechens“ als eine Form des freiwilligen, beiderseits vorteilhaften Tausches legitimiert. Die ,harten` ökonomischen und sozialen Machtasymmetrien, die aus unterschiedlichen Vermögenspositionen resultieren, werden also in gramscianischer Manier um ,weiche' oder ,hegemoniale‘ Momente der Zustimmung ergänzt, um die gesellschaftlichen Verhältnisse zu stabilisieren (siehe bereits Macchiavelli, 1532 oder Gramsci, 1927-1935).

In modernen Gesellschaften ortet Piketty ein „neo-proprietaristisches Eigentumsregime“, das auf einer heuchlerischen, aber nichtsdestotrotz erfolgreichen meritokratischen Erzählung beruht, in der Eigentum Anreize sichert und der marktliche Wettbewerb garantiert, dass Leistungen und Beiträge einander weitgehend entsprechen. Beides - Marktfreiheiten und erworbene Eigentumsrechte dürfen daher nicht in Frage gestellt oder zu stark politisch reguliert werden. Piketty zeichnet die praktischen Folgen dieser Erzählung vor allem für den konkreten Zusammenhang der Steuerpolitik detailliert nach, indem er auf das Beispiel der stark progressiven Einkommens- und Vermögenssteuern fokussiert. Für Piketty waren diese nicht nur eine zentrale Ausgangsbedingung für die Etablierung eines „sozialdemokratischen" Zeitalters in den Jahrzehnten nach dem zweiten Weltkrieg, sondern sie bilden auch den wesentlichen Stellriemen zur Etablierung des „Neo-Proprietarismus“, der sich - zusätzlich befeuert durch den internationalen Standortwettbewerb einer „unzureichend kollektiv regulierten Globalisierung“ (Piketty: 936) - in der Abschaffung von vermögensbezogenen Steuern ebenso niederschlägt wie in einer generell sinkenden Progression der vorherrschenden Steuersysteme. 
Zur näheren Erläuterung der Unterschiede und Eigenschaften verschiedener Ungleichheitsregime unterscheidet Piketty zwischen einem politischen Regime der Grenze (also der grundsätzlichen territorialen und politischen Ordnung des Nationalstaats) und einem Eigentumsregime (das Fragen der Rechtmäßigkeit von Eigentum, Besteuerung und dergleichen klärt). Die Entwicklung dieser beiden Ordnungen ist meist eng aneinandergekoppelt, wie Piketty am Beispiel einer international-vergleichenden Darstellung der Geschichte des Wahlrechts eindrucksvoll illustriert. Schließlich verortet er am Ende des Buches die zentralen gesellschaftlich-politischen Bruchlinien der entwickelten Gesellschaften des 21. Jahrhundert entlang dieser beiden Dimensionen.

Piketty entwirft wie schon in „Kapital im 21. Jahrhundert“ in einer quasi-induktivistischen Herangehensweise eigene Begriffswelten, die versuchen, die aus den empirischen Daten hervorgehenden Muster und Eigenschaften möglichst zusammenzufassend zu beschreiben. Diese Methodik, die sich wenig um etablierte Sichtweisen und Terminologien kümmert und an vielen Stellen einer quantitativen Interpretation eines grounded theory Ansatzes zu entsprechen scheint, hat eine Reihe von Vorteilen: sie erlaubt einen frischen Blick auf den Gegenstand, ermöglicht eine nuancierte Debatten der historischen Vorgänge und erlaubt eine sehr datengetriebene und reflektierte Präsentation der eigenen Vorstellungen. Auffällig ist in diesem Zusammenhang, dass Piketty kaum auf die in „Kapitel im 21. Jahrhundert“ aufgebauten Metaphern (wie den „patrimonialen Kapitalismus“, das „Rastignac Dilemma“ oder das „Kapital-Einkommens-Verhältnis“) zurückgreift, sondern die theoretische Rahmung neu setzt und somit das Historische, Soziologische und Politikwissenschaftliche - kurz: das Gesellschaftswissenschaftliche - gegenüber dem genuin Ökonomischen in seinem Narrativ weiter stärkt. Allerdings bleibt die Frage offen, ob und in welchem Ausmaß eine solche Theoriebildung, die nur vereinzelt versucht, einen Bezug zu etablierten Theorien herzustellen, im wissenschaftlichen Diskurs Anschluss findet. Dies ist insbesondere deshalb ein Versäumnis, weil sich Piketty an ein interdisziplinäres Publikum richtet, es aber so erschwert, seine durchaus nennenswerten Einsichten in bestehende Diskurse einzubinden.

„Kapital und Ideologie“ richtet sich somit an ein interessiertes Laienpublikum; es ist ein umfassendes Sachbuch, nicht ein wissenschaftliches Buch im engeren Sinne. Ein wissenschaftlicherer Anspruch käme nicht ohne die Zitierung kontemporärer soziologischer, politikwissenschaftlicher und ökonomischer Analysen aus, die in einzelnen Bereichen naturgemäß stärker in die Tiefe gehen, als Piketty es in diesem seinem zweiten Opus Magnum zu tun vermag. Das Verdienst Pikettys in „Kapital und Ideologie“ liegt somit darin, dass er sich den etablierten Wissensstand in weiten Teilen von Neuem erarbeitet und dabei innovative Schwerpunkte setzt, die sich auch prospektiv als fruchtbringend erweisen. 
So ergänzt Piketty die traditionellen Vorstellungen einer „internationalistisch-egalitären Linken“ und der „nationalistisch-inegalitären Rechten“ um eine „Brahmin Left“, die ihre WählerInnenschaft stärker in der Bildungselite als in der ArbeiterInnenklasse hat und dabei tendenziell internationalistisch und inegalitär agiert, und eine „sozial-nativistische“ Bewegung, die neben abschottenden und anti-emanzipatorischen Zügen zumindest in ihrer Rhetorik auf nationale Egalität setzt. Diese Erweiterung kann etwa erklären, warum WählerInnen im Zuge der US-amerikanischen Präsidentschaftswahlen 2020 im republikanischen Texas für einen höheren Mindestlohn votierten, während im demokratischen Kalifornien die Stärkung von ArbeitnehmerInnen-Rechten in Plattformökonomien (z. B. Uber) abgelehnt wurde. Auch andere Phänomene in der jüngeren Entwicklung etablierter Demokratien - wie die Pulverisierung der französischen Parti Socialiste, das britische Brexit-Votum, der Aufstieg der AfD oder die Dominanz der hindu-nationalistischen BJP in Indien - fügen sich harmonisch in Pikettys allgemeines Erklärungsschema ein.

\section{Thomas Piketty als Forscher und, Public Intellectual'}

Mit „Kapital und Ideologie“ vollzieht Piketty eine seit geraumer Zeit absehbare Wandlung, in der er sich zu einem sozialwissenschaftlichen Universalgelehrten entwickelt, dessen Theorie antike Sklavengesellschaften ebenso umfasst wie das indische Kastensystem oder die modernen Hocheinkommensgesellschaften. Mit „Kapital im 21. Jahrhundert“ war es Piketty gelungen, als arrivierter Ökonom aus einer Position der Mitte Verteilungsthemen vom Rand ins Zentrum der ökonomischen Debatte zu holen. In „Kapital und Ideologie“ versucht er dagegen nicht mehr, sein Fach von der Bedeutung von Ungleichheit oder dem Mehrwert akribisch-empirischer wirtschaftshistorischer Arbeit $\mathrm{zu}$ überzeugen, sondern richtet sich vielmehr an die citoyen(ne)s, die gemeinsam Welt und Gesellschaft gestalten können.

Tatsächlich enthält das Buch eine Reihe von Elementen, die selbiges aus standardökonomischer Sicht unbrauchbar erscheinen lassen: Pikettys historisch-induktiver Fokus, die Absenz von Referenzen auf die Standardlehrbuchökonomie, seine explizite Positionierung als Anhänger eines „partizipativen Sozialismus“ oder die Darstellung von Ungleichheit - anstelle von Effizienz - als zentrales und konstitutives Merkmal des Kapitalismus. All dies sind Elemente, mit denen sich Piketty jenseits der fachkulturellen Grenzen der Standardökonomie positioniert. Während die progressive Mainstream-Ökonomie „Kapital im 
21. Jahrhundert“" wohlwollend aufnahm - herausragende Unterstützer waren etwa Branko Milanovic, Paul Krugman oder Joseph Stiglitz -, fällt er mit seiner neuerdings sozialwissenschaftlicheren Zugangsweise aus der ökonomischen Diskussion zunehmend heraus: Milanovic (2019) etwa kritisiert vorsichtig die überambitionierte historische Untersuchung, aber schätzt die Analyse sozialdemokratischer Parteien im vierten Teil des Buches. Krugman (2020) dagegen schreibt in der New York Times, Piketty habe „Marx auf den Kopf gestellt“ und dem Buch fehle eine klare Botschaft. Im Großen und Ganzen wird „Kapital und Ideologie“ allerdings innerhalb der Ökonomik mit Ignoranz gestraft.

Piketty seinerseits beraubte sein Oeuvre bereits in „Kapital im 21. Jahrhundert“ um Referenzen auf marxistische, keynesianische und regulationstheoretische Ansätze, die teils großen Vorsprung in der Untersuchung von Verteilungsfragen gegenüber der neoklassischen Theorie haben, auf welche sich Piketty stützte. Obgleich sichtlich von der französischen Annales Schule beeinflusst, verweist Piketty auch in „Kapital und Ideologie“ nur eingeschränkt auf historische, soziologische und politikwissenschaftliche Beiträge. Die Rezeption von „Kapital und Ideologie“ scheint bisher von dieser Seite daher ebenso verhalten. Der vielleicht prominenteste Review von Pikettys neuestem Buch erschien von einer jungen progressiven Ökonomin, Ingrid Harvold Kvangraven (2020), in der Fachzeitschrift Nature. Sie nennt das Buch „zeitgemäß aber inadäquat“ und kritisiert insbesondere die fehlende Wahrnehmung Pikettys bezüglich der ,politischen Auseinandersetzungen um Ideen an den Universitäten [...] einschließlich des Verdrängens keynesianischer und marxistischer Perspektiven an den Ökonomie-Fakultäten“ - ein Problem, das Piketty tatsächlich umschifft und erst in seinem Schlusswort verklausuliert anzusprechen vermag. Pikettys Sicht seiner empirischen Datenarbeit als „rational“ und „,neutral“ (im Gegensatz zu seinen „ideologischen“ Politikempfehlungen) perpetuiere dieses Problem.

„Kapital und Ideologie“ reiht sich mit seinem Plädoyer an die BürgerInnen in Pikettys mit Weitblick gestellte strategische Weichen nach der Erfolgswelle von „Kapital im 21. Jahrhundert“ ein: er nimmt aktiver an tagespolitischen Debatten teil und setzt auf gezielte Nachwuchsförderung an der Paris School of Economics sowie den Ausbau der Grundlagenforschung. Letzteres treibt er mittels der World Inequality Database voran, die Ungleichheitsdaten sowie -reports publiziert und die er auch in „Kapital und Ideologie“ prominent platziert. Zudem entwickelte Piketty mit seinem ehemaligen Schüler Gabriel Zucman und mit Emmanuel Saez die sogenannten „Distributional National Accounts“ (DINA), die Verteilungsinformationen in administrative Daten einspeisen sollen. In beiden Fällen entstand ein reges Forschungsprogramm, das jungen VerteilungsforscherInnen die Möglichkeit bietet, ihre Forschung in international renommierten Fachzeitschriften zu veröffentlichen. Auf wirtschaftspolitischer Ebene veröffentlichte Piketty einen 
„Appell für ein anderes Europa“ und unterstützte zeitweise progressive Gruppen und Bewegungen, darunter etwa Jeremy Corbyn und Benoit Hamon.

\section{Ausblick: Thomas Piketty als humanistischer Empirist}

Auch auf der Ebene der politischen Implikationen und Schlussfolgerungen bleibt Piketty seiner grundsätzlichen Linie treu, die politische Forderungen nicht aus theoretischen Überlegungen deduziert, sondern ihre Inspiration vielmehr aus dem Kontrast zwischen empirischen Material und humanistischen Wertvorstellungen zieht. Diese Vorgehensweise ergibt sich für Piketty aus der Einsicht, dass die soziale Welt durch Staaten, Rechtsnormen und Steuern gezielt gestaltet werden kann und de facto auch immer auf Basis von Wertvorstellungen gestaltet wird. Diese Gestaltungsfragen als Wertfragen zu deklarieren, ist für Piketty daher nicht nur intellektuell konsequent - man kann es auch als Versuch verstehen, vermeintliche politische Sachzwänge zu demaskieren und als primär interessensgeleitete Konstruktionen zu entlarven.

Zum Abschluss seines Buchs entwickelt Piketty ein Modell eines „partizipativen Sozialismus“, der im Kern auf stark progressiven Einkommens- und Vermögenssteuern beruht, die zu einer de facto Obergrenze von Einkommen und Vermögen führen und eine Form des „Eigentums auf Zeit“ etablieren sollen, um endogenen Konzentrationstendenzen vorzubeugen. Dazu gesellt sich ein breiter Blumenstrauß egalisierender und demokratiestärkender Maßnahmen, die von der Beteiligung der ArbeitnehmerInnen in den Unternehmensleitungen über eine massive Ausweitung des Zugangs zu tertiärer Bildung bis hin zu einer Neugestaltung der europäischen Institutionen mit dem Ziel der Beendigung des innereuropäischen Steuer- und Standortwettbewerbs reicht. Beeindruckend ist dabei, dass die von Piketty vorgestellten Maßnahmen in hohem Maße konsistent erscheinen hier wird das Buch seinem Anspruch, BürgerInnen zu ermächtigen, tatsächlich vollumfänglich gerecht: Dass die grundsätzliche Systemkritik sowie die Formulierung echter Alternativen zum status quo in „Kapital und Ideologie“ ohne großen theoretischen Ballast auskommt, macht diese Aspekte hochgradig alltagstauglich und bietet die Chance, politische Diskurse nachhaltig zu beeinflussen - zumindest unter der Annahme, dass auch wirklich genug LeserInnen bis zum Ende des Buches durchhalten.

Danksagung: Wir danken Mathias Johmetzer für wertvolle Hinweise. Alle verbleibenden Fehler liegen in unserer Verantwortung. 


\section{Literatur}

Cohen, A. J.; Harcourt, G. C. Retrospectives Whatever Happened to the Cambridge Capital Theory Controversies? Journal of Economic Perspectives 2003, 17, 199-214.

Gramsci, A. Gefängnishefte; Argument-Verlag: Berlin, 1927-1935.

Harvold Kvangraven, I. Post-Pandemic Overhaul will take more than Tweaks. Nature 2020, 580, 582-583.

Krugman, P. Thomas Piketty Turns Marx on His Head. New York Times 2020

https://www.nytimes.com/2020/03/08/books/review/capital-and-ideology-thomas-

piketty.html (Zugriff Dez 10, 2020).

Machiavelli, N. Der Fürst; Kröner Verlag: Stuttgart, 1978 [1532].

Milanovic, B. Global Inequality. A New Approach for the Age of Globalization; Belknap Press: Cambridge, Mass., 2016.

Milanovic, B. Thomas Piketty's New Book Brings Political Economy Back to its Sources. Promarket 2019 https://promarket.org/2019/09/06/thomas-piketty-new-book-brings-politicaleconomy-back-to-its-sources/ (Zugriff Dez 10, 2020).

Mill, J. S. Principles of Political Economy with some of their Applications to Social Philosophy; Longmans, Green and Co: London, 1902 [1848].

Scheidel, W. The Great Leveler: Violence and the History of Inequality from the Stone Age to the Twenty-First Century; Princeton University Press: Princeton, New Jersey, 2017.

van Bavel, B. The Invisible Hand? How Market Economies have Emerged and Declined since 500 $A D$; Oxford University Press: Oxford, 2016. 